\title{
Platelet Activation Is Limited during Transcatheter Aortic Valve Implantation in Patients on Aspirin Monotherapy and without per Procedural Clinical Complications
}

\author{
Nicolas Dumonteil ${ }^{1, *}$ Marie Levade ${ }^{2,3, *}$ Cédric Garcia $^{3}$ Thibault Lhermusier ${ }^{1,2}$ Jennifer Series ${ }^{3}$ \\ Pauline Le Faouder $^{4}$ Bertrand Marcheix ${ }^{1}$ Bernard Payrastre ${ }^{2,3}$ Didier Carrié ${ }^{1,2}$ Pierre Sié2,3 \\ ${ }^{1}$ Pôle Cardiovasculaire et Métabolique, Hôpital Rangueil, Toulouse, \\ France \\ 2 INSERM, U1048 and Université Toulouse 3, Institut des Maladies \\ Address for correspondence Pierre Sie, MD, PhD, Lab Hématologie, \\ CHU Rangueil, 1 Avenue du Pr Jean Poulhès, TSA 50032, 31059 \\ Métaboliques et Cardiovasculaires, Toulouse, France \\ Toulouse Cedex 9, France (e-mail: sie.p@chu-toulouse.fr). \\ ${ }^{3}$ Laboratoire d'Hématologie, Centre Hospitalier Universitaire de \\ Toulouse, Toulouse, France \\ ${ }^{4}$ MetaToul-Lipidomique, INSERM U1048 and Université Paul Sabatier \\ Toulouse 3, Toulouse, France \\ TH Open 2019;3:e146-e152.
}

\begin{abstract}
Keywords

- platelet activation

- aortic valve stenosis

- transcatheter aortic valve implantation
\end{abstract}

Transcatheter aortic valve implantation (TAVI) is an established treatment option for symptomatic patients with severe aortic valve stenosis (AS). During and early after the procedure, both ischemic events (predominantly stroke) and bleedings remain prevalent. The optimal antithrombotic regimen is still debated. Single- versus dualantiplatelet therapy is associated with a lower rate of severe bleeding, without difference in thrombotic complications. Although platelets have been empirically targeted, little is known on their contribution to these events primarily related to embolization of thrombotic material and tissue-derived debris from the wounded aortic valve and large vessels. The objective of this study was to assess local platelet activation in blood sampled in the ascending aorta immediately before and within minutes postimplantation. A series of 18 patients with AS on monotherapy with aspirin successfully underwent TAVI with the self-expandable Medtronic CoreValve by transfemoral route. No clinical thrombotic complication occurred at 30-day followup. Compared with patients with stable coronary artery disease unscathed of AS and similarly treated by low-dose aspirin, AS patients displayed a chronic state of platelet activation before TAVI, assessed in venous blood using various biomarkers. However, per procedure, in aortic blood, no change occurred between the two time points in the plasma levels of serotonin or 12-lipoxgenase products, or membrane exposure of granule markers CD62-P and CD63. Our results suggest that local acute platelet activation is limited during TAVI on monotherapy with aspirin.

* These authors contributed equally to this study.

received

November 5, 2018 accepted after revision April 18, 2019
DOI https://doi.org/

10.1055/s-0039-1692142. ISSN 2512-9465. (c) 2019 Georg Thieme Verlag KG Stuttgart · New York
License terms

(c) (i) 


\section{Introduction}

Transcatheter aortic valve implantation (TAVI) is currently the standard of care for the treatment of symptomatic severe aortic valve stenosis (AS) for patients with high surgical risk. ${ }^{1}$ In spite of improvement of devices and operators' experience and better patient assessment during the last decade, TAVI still carries a significant periprocedural thromboembolic and concomitant bleeding risk ${ }^{2}$ that fuels the debate on the optimal antithrombotic therapy in patients undergoing TAVI. ${ }^{3,4}$ Although clinically apparent cerebral ischemia and persistent neurological impairment rarely occur, the incidence of clinically silent cerebral embolic lesions after TAVI is high. Most strokes occur in the acute phase (first day) and are strongly related to procedural factors. ${ }^{3}$ Transcranial Doppler has demonstrated the role of interaction of the device with the native aortic valve as the main cause of cerebral embolization. ${ }^{5}$ Captured debris in filters deployed in large cerebral arteries during the procedure associate thrombotic material to tissue-derived debris. ${ }^{6}$ However, the mechanical stress to the aorta and to the calcified native valve caused by catheter manipulation, balloon dilation, retrograde valve positioning, and frame expansion induces a high thrombogenic surface that could strongly activate the platelets and the coagulation pathways until endothelialization. Although the contribution of platelets to acute thromboembolic events may be a potential important issue, it has never been directly investigated. To investigate this point, we have measured local platelet activation in blood sampled in the ascending aorta immediately before and within minutes postimplantation before removing the catheter, in patients undergoing TAVI. Platelet activation in aortic whole blood was assessed by measurement of secretion of serotonin and bioactive lipids and expression of membrane granule markers and compared between the two time points. All patients were on monotherapy with aspirin according to our local protocol based on a low rationale for dual-antiplatelet pretreatment before TAVI. ${ }^{7}$ The primary laboratory endpoint of the study was the comparison of platelet activation markers in aortic whole blood sampled pre- and immediately post-TAVI. The secondary laboratory endpoint was the comparison of platelet activation markers in peripheral venous blood before TAVI between patients with severe aortic stenosis (AS group) and patients with stable coronary artery disease (CAD) without aortic stenosis (CAD group).

\section{Methods}

\section{Patients}

This prospective, monocentric, observational study (NCT02504632) was performed in 20 high-risk patients with severe symptomatic AS (aortic valve area [AVA] $<1 \mathrm{~cm}^{2}$ or $0.6 \mathrm{~cm}^{2} / \mathrm{m}^{2}$ of body surface area) undergoing TAVI for indications according to current recommendations. ${ }^{8,9}$ Patients were not included if they had acute coronary syndrome 1 month before inclusion, terminal chronic kidney disease requiring hemodialysis, baseline indication of dual-antiplatelet therapy (DAPT) or current anticoagulant treatment, thrombocytopenia less than $100 \mathrm{G} / \mathrm{L}$, or hemoglobin less than $100 \mathrm{~g} / \mathrm{L}$. The patients received a monotherapy by aspirin $(75-160 \mathrm{mg} / \mathrm{d}$ ) for at least 1 week before TAVI.

A group of 26 patients admitted for coronary angiogram assessment for stable CAD served as comparator. These patients were unscathed of AS, based on cardiac echography performed mainly as outpatients in the months before, and on clinical exam at admission.

The research protocol complies with the Declaration of Helsinki and was approved by the Toulouse University Hospital Human Research and Ethics Committee. Informed consent was obtained from all participants.

\section{Transcatheter Aortic Valve Implantation Procedure}

The third-generation Medtronic CoreValve ReValving System (Medtronic, Minneapolis, Minnesota, United States) was implanted by the same operator in all patients by transfemoral approach with double Pro-Glide (Abbott Vascular, Redwood City, California, United States) preclosing, as previously published. ${ }^{10}$ Procedures were performed under anticoagulation by a bolus of unfractionated heparin at weight-adjusted dose.

The clinical endpoints after TAVI were described following Valve Academic Research Consortium-2 definitions. ${ }^{11}$ Inhospital clinical, biological, and transthoracic echocardiography (TTE) follow-up was performed before discharge. Active 30-day follow-up was obtained in all survivors by outpatient visit or direct contact with their cardiologist. All events and values were prospectively site recorded.

\section{Blood Collection and Preparation of Samples}

Aortic blood was sampled into $0.109 \mathrm{M}$ sodium citrate through the pig-tail catheter downstream aortic valve, just before (T1) and 10 to 15 minutes after (T2) the implantation of the prosthesis. A venous blood sample was collected by forearm venepuncture at the beginning of the procedure, before heparin administration, and manipulation of the aortic valve. Platelet-rich plasma (PRP) and platelet-poor plasma (PPP) were immediately obtained by differential centrifugation. PPP for measurement of soluble markers and PPP mixed with cold methanol (1:1, v:v) for eicosanoid quantification were stored at $-80^{\circ} \mathrm{C}$, whereas platelet membrane markers of granule secretion were assessed on fresh PRP.

\section{Laboratory Methods}

Commercial enzyme-linked immunosorbent assays were used for quantification of soluble markers such as soluble platelet glycoprotein VI (sGPVI; MyBioSource, San Diego, California, United States), sP-selectin, and sCD40-ligand (eBioscience, Paris, France) and serotonin (IBL-America, Minneapolis, Minnesota, United States) in PPP. Platelet aggregation in response to $5 \mu \mathrm{M}$ adenosine diphosphate (ADP, Sigma), $50 \mu \mathrm{M}$ thrombin receptor activating peptide (TRAP, Sigma), or $10 \mu \mathrm{g} / \mathrm{mL}$ collagen-related peptide (CRP, from Prof. Farndale, University of Cambridge, UK) was studied in PRP using light transmission aggregometry (TA-8V aggregometer, SD Medical). Results are expressed in \% maximal 
aggregation (reference laboratory values: 70-100\%). Platelet granule secretion was assessed by measuring transmembrane glycoproteins CD62-P and CD63, markers of $\alpha$ and $\delta$ granules, respectively, by flow cytometry. PRP was incubated with anti-CD62P FITC or anti-CD63 FITC-conjugated antibodies (BD Pharmingen, BD Biosciences, Le Pont-de-Claix, France) for 15 minutes at room temperature in the dark. The analysis was performed using a BD FACS Verse cytometer (BD Biosciences). Results were expressed as median fluorescence intensity (MFI) for unstimulated platelets (upper normal limit: 40 arbitrary units), or as MFI-fold increase over resting value in PRP stimulated with $10 \mu \mathrm{g} / \mathrm{mL}$ CRP or $50 \mu \mathrm{M}$ TRAP (Sigma-Aldrich, Saint-Quentin-Fallavier, France) for 10 minutes at $37^{\circ} \mathrm{C}$.

Plasma eicosanoids were quantified by liquid chromatography-tandem mass spectrometry (LC-MS/MS) as previously described. ${ }^{12}$ Internal standard containing LxA4-d5, LtB4-d4, and 5-HETE-d8 at $400 \mathrm{ng} / \mathrm{mL}$ (Cayman Chemicals, Ann Arbor, Michigan, United States) was added to the plasma/methanol before lipid extraction. Lipoxins A4 and B4; resolvins D1, D2, and 7(S)-maresin; leukotrienes B5, 10 (S), and 17(S)-protectin; 9- and 13-hydroxyoctadecadienoic acids; 5-, 12-, and 15-hydroxyeicosatetraenoic acids (HETE); and 14- and 17-hydroxydocosahexaenoic acid (HDoHE) were separated on a ZorBAX SB-C18 column using Agilent 1290 Infinity HPLC system coupled to an ESI-triple quadruple G6460 mass spectrometer (Agilent Technologies, Santa Clara, California, United States). Data were acquired in Multiple Reaction Monitoring mode with optimized conditions (ion optics and collision energy). Peak detection, integration, and quantitative analysis were done using Mass Hunter Quantitative analysis software (Agilent Technologies) based on calibration lines built with internal standards.

\section{Study Endpoints}

The clinical endpoints after TAVI were described following Valve Academic Research Consortium definitions. ${ }^{11}$ In-hospital clinical, biological, and TTE follow-up was performed before discharge. Thirty-day follow-up was active and was obtained in all survivors by medical visit or direct contact with their cardiologist. All events and values were prospectively site recorded.

The primary laboratory endpoint was the comparison of platelet activation markers in aortic whole blood sampled pre- and immediately post-TAVI. The secondary laboratory endpoints were the comparison before TAVI of platelet activation markers in peripheral venous blood with the values measured in aortic blood at $\mathrm{T} 1$ and with venous blood of CAD patients.

\section{Statistical Analysis}

Sample size was calculated according to the primary objective of the study, that is, to assess local platelet activation in aortic blood within minutes following prosthetic valve implantation (T2).

In static conditions, in vitro platelet secretion induced by soluble agonists produces a large increase of cytokines or bioactive lipids released in plasma. We assumed that, if $\sim 10 \%$ of circulating platelets were activated by passing through the valve, the levels of plasma markers will double in the aortic blood sampled close to the injured vessel. Given this assumption and the standard deviations provided for the various hemostasis parameters in preliminary analyses, we calculated that 20 subjects would be needed to assess such a doubling of hemostasis parameters between $\mathrm{T} 1$ (before the procedure) and T2, under the hypothesis of a $90 \%$ power, a $5 \%$ type 1 error rate, and using a paired nonparametric test.

Statistical analysis was performed using GraphPad Prism software. Continuous variables were expressed as mean \pm standard deviation, or median (interquartile range [IQ]: 25th-75th) as appropriate, and compared between groups or between time points using the nonparametric MannWhitney or paired Wilcoxon's tests. Multilinear regression analysis with age and group was performed on matched population. Discrete variables were expressed as number and percentage, and compared using the Fisher test.

\section{Results}

\section{Baseline Characteristics of Patients and Procedural Clinical Outcomes}

The results of two included patients with AS were removed for technical reasons. The baseline characteristics of the other 18 patients with AS are depicted in - Table 1. None of them had bleeding symptoms before the procedure. The success rate of VARC-2 device was 100\%. Within the first 24 hours, platelet counts significantly dropped by $23 \%$ from $214 \pm 71$ to $165 \pm 51 \mathrm{G} / \mathrm{L}$, and hemoglobin by $12 \%$ from $121 \pm 15$ to $107 \pm 17 \mathrm{~g} / \mathrm{L}$, whereas a transient increase in leukocyte count occurred, from $7.4 \pm 2.1 \mathrm{G} / \mathrm{L}$ to $9.4 \pm 2.6 \mathrm{G} / \mathrm{L}$. One death related to a cardiac tamponade occurred few hours after the procedure. The other 17 patients reached the 30-day follow-up visit alive, with a functional improvement and a good function of the aortic bioprosthesis (mean AVA: $1.9 \pm 0.4 \mathrm{~cm}^{2}, 16$ patients with none or trace and 1 with mild paravalvular regurgitation on TTE).

The group of 26 patients with stable CAD were significantly younger, have no cardiac failure above NYA class II, and by definition most of them had CAD confirmed on angiography (-Table 1). Creatinine and hemoglobin levels were, respectively, lower and higher in the CAD group than in the AS group.

\section{Platelet Activation Markers in Venous Blood before TAVI Procedure}

Compared with patients with stable CAD, the plasma levels of biomarkers of platelet activation, sGPVI and SCD40L, shed from the platelet membrane, and products of platelet lipid metabolism, were significantly higher in the AS group. However, after multivariate regression analysis, using age and groups as covariable, the difference was nonsignificant for SGPVI and SCD40L. Among the different bioactive lipids analyzed, we focused on the main products of 12-lipoxygenase (LOX), 12-HETE, and 14-HDoHE, released in plasma upon platelet activation. These two lipids were significantly correlated ( $\left.r^{2}=0.66, p=0.007\right)$. As our patients were taking 
Table 1 Key baseline demographic and clinical data of patients in AS before TAVI and CAD groups

\begin{tabular}{|c|c|c|c|}
\hline & AS $(n=18)$ & CAD $(n=26)$ & $p$ \\
\hline Age $(y)$, mean $\pm S D$ & $86 \pm 4$ & $68 \pm 12$ & $<10^{-4}$ \\
\hline Male, $n(\%)$ & $10(55)$ & $19(73)$ & 0.53 \\
\hline Body mass index, mean $\pm S D$ & $25 \pm 4$ & $27 \pm 6$ & 0.23 \\
\hline New York Hearth Association class III or IV, $n$ (\%) & $15(83)$ & 0 & $<10^{-4}$ \\
\hline Logistic EuroSCORE, mean \pm SD & $21 \pm 11$ & NA & \\
\hline Coronary artery disease, $n(\%)$ & $12(66)$ & $24(92)$ & 0.048 \\
\hline Diabetes mellitus, $n(\%)$ & $4(22)$ & $9(34)$ & 0.34 \\
\hline Hypertension, $n(\%)$ & $12(66)$ & $18(69)$ & 1.0 \\
\hline Chronic obstructive pulmonary disease, $n$ (\%) & $3(16)$ & $1(4)$ & 0.29 \\
\hline Peripheral vascular disease, $n(\%)$ & 0 & $6(23)$ & 0.07 \\
\hline Creatinine $(\mu \mathrm{mol})$, mean $\pm \mathrm{SD}$ & $125 \pm 53$ & $93 \pm 22$ & 0.011 \\
\hline Hemoglobin $(\mathrm{g} / \mathrm{L})$, mean $\pm \mathrm{SD}$ & $121 \pm 15$ & $141 \pm 13$ & $<10^{-3}$ \\
\hline Platelet count $(\mathrm{G} / \mathrm{L})$, mean $\pm \mathrm{SD}$ & $214 \pm 71$ & $254 \pm 70$ & 0.081 \\
\hline \multicolumn{4}{|l|}{ Echocardiography, mean \pm SD } \\
\hline Aortic valve area $\left(\mathrm{cm}^{2}\right)$ & $0.77 \pm 0.11$ & NA & \\
\hline Left ventricular ejection fraction (\%) & $51.7 \pm 11.9$ & NA & \\
\hline Mean pressure gradient $(\mathrm{mm} \mathrm{Hg})$ & $43.3 \pm 14.5$ & NA & \\
\hline
\end{tabular}

Abbreviations: AS, aortic valve stenosis; CAD, coronary artery disease; NA, not available; SD, standard deviation; TAVI, transcatheter aortic valve implantation.

Note: Continuous variables were compared using the nonparametric Mann-Whitney test and discrete variables using Fisher's test.

aspirin, cyclooxygenase (COX) products, especially thromboxane B2, were undetectable. The levels of other detectable lipid products on the LC-MS/MS profile (5-HETE, 15-HETE, 9-HODE, and 13-HODE), mainly produced by leukocytes and endothelial cells, were not significantly different between the two patient groups (not shown). Plasma levels 12-HETE and 14-HDoHE remained significantly higher in AS group compared with CAD after adjustment for age $(p=0.003$ and 0.007, respectively). Membrane exposure of CD62-P and CD63 by unstimulated platelets was low in both patient groups, with MFI in the normal reference range of the laboratory. Platelet aggregation (maximal amplitude) induced by ADP, CRP, and TRAP in PRP was in the low normal range in both groups, as usually found under aspirin therapy. Only CRP-induced aggregation was slightly, but significantly, lower in AS patients, possibly due to loss of membrane GPVI. Membrane exposure of CD62P and CD63 was induced by CRP or TRAP at similar extent in both groups. These results suggest that platelets circulating in peripheral blood are not desensitized by multiple passages through the stenotic valve and that, in static conditions, circulating platelets were reactive to soluble agonists ( - Table $\mathbf{2}$ ).

\section{Local Platelet Activation in Aortic Blood during TAVI Procedure}

To catch platelet activation on the surface of injured valve, we measured platelet activation in blood sampled in the ascending aorta immediately before (T1) and within minutes postimplantation, before removing the catheter (T2). At T1, the levels of bioactive lipids $\left(13.7^{[6.0-32.2]} \mathrm{ng} / \mathrm{mL}\right.$ for 12 -HETE and
$3.5^{[1.9-7.5]} \mathrm{ng} / \mathrm{mL}$ for 14 -HDoHE, median ${ }^{[\mathrm{QQ} 25-75]}$ ) were nonsignificantly different from those measured in venous blood at the same time (see - Table 2, $p=0.74$ and 0.38 , respectively, paired Wilcoxon's test). Similarly, membrane exposure of CD62-P or CD63 by resting platelets was low. This rules out platelet activation induced by blood collection through the transfemoral catheter or induced by unfractionated heparin at therapeutic concentrations administered before the procedure. ${ }^{13}$

As shown in - Fig. 1A-C, 12-HETE, 14-HDoHETE, and serotonin demonstrated no significant change between the two time points. As expected for two products of the same metabolic pathway, 12-HETE and 14-HDoHE levels were linearly correlated, both at T1 $\left(r^{2}=0.40, p<10^{3}\right)$ and T2 $\left(r^{2}=0.59, p<10^{3}\right)$. In contrast, they were not correlated with serotonin levels at any time $(p=0.89$ and 0.93 , respectively). Membrane exposure of CD62-P (MFI ${ }^{[\mathrm{IQ} 25-75]}: 33^{[16-57]}$ and $38^{[11-67]}$ before and after, respectively, $p=0.67$ ), or CD63 $\left(27^{[23-32]}\right.$ and $\left.30^{[17-32]}, p=0.36\right)$ did not vary and remained low.

\section{Discussion}

The association between shear stress and hemostatic activation has been demonstrated in vitro and in vivo in various clinical conditions, severe aortic stenosis being one of the best documented. Increased levels of SCD40L or SGPVI ${ }^{14,15}$ in conjunction with a reduction of surface GPIba or GPVI, ${ }^{16}$ platelet-derived chemokines ${ }^{17}$ and $\operatorname{serotonin}^{18}$ in platelet-free plasma, or of circulating platelet-derived 
Table 2 Baseline platelet activation markers and in vitro platelet reactivity in AS before TAVI and control groups

\begin{tabular}{|c|c|c|c|c|}
\hline \multicolumn{2}{|l|}{ Platelet activation markers in venous blood } & AS $(n=18)$ & $\operatorname{CAD}(n=26)$ & $p$ \\
\hline \multicolumn{2}{|l|}{ Soluble CD40 ligand (ng/mL), median $\left[\mathrm{IQ}^{25-75}\right]$} & $3.3^{[0.9-6.6]}$ & $1.0^{[0.6-1.4]}$ & 0.027 \\
\hline \multicolumn{2}{|l|}{ Soluble P-selectin $(\mathrm{ng} / \mathrm{mL})$, median $\left[\mathrm{IQ}^{25-75}\right]$} & $69^{[56-97]}$ & $61^{[48-86]}$ & 0.30 \\
\hline \multicolumn{2}{|l|}{ Soluble GPVI (ng/mL), median [IQ $\left.{ }^{25-75}\right]$} & $58^{[26-80]}$ & $16^{[5-44]}$ & 0.026 \\
\hline \multicolumn{2}{|l|}{ 12-HETE (ng/mL) median $\left[\mathrm{IQ}^{25-75}\right]$} & $9.0^{[7-21]}$ & $2.6^{[1.5-3.9]}$ & $<10^{3}$ \\
\hline \multicolumn{2}{|l|}{ 14-HDoHE (ng/mL) median [IQ $\left.{ }^{25-75}\right]$} & $2.7^{[1.9-7.3]}$ & $0.7^{[0.4-0.9]}$ & $<10^{3}$ \\
\hline $\begin{array}{l}\text { Maximal amplitude of platelet aggregation in } \\
\text { PRP, mean } \pm \text { SD }\end{array}$ & $\begin{array}{l}\text { ADP } 5 \mu \mathrm{M} \\
\text { CRP } 10 \mu \mathrm{g} / \mathrm{mL} \\
\text { TRAP } 50 \mu \mathrm{M}\end{array}$ & $\begin{array}{l}58 \pm 16 \\
66 \pm 11 \\
71 \pm 11\end{array}$ & $\begin{array}{l}58 \pm 19 \\
80 \pm 10 \\
75 \pm 9\end{array}$ & $\begin{array}{l}0.8 \\
<0.01 \\
0.5\end{array}$ \\
\hline \multicolumn{2}{|l|}{$\begin{array}{l}\text { CD62-P surface exposure (MFI) on resting platelets, } \\
\text { median }\left[1 \mathrm{Q}^{25-75}\right]\end{array}$} & $35^{[12-59]}$ & $25^{[9-46]}$ & 0.25 \\
\hline \multicolumn{2}{|l|}{$\begin{array}{l}\text { CD63 surface exposure (MFI) on resting platelets, } \\
\text { median }\left[\mathrm{IQ}^{25-75}\right]\end{array}$} & $26^{[20-28]}$ & $23^{[20-54]}$ & 0.70 \\
\hline \multirow{2}{*}{$\begin{array}{l}\text { CD62-P MFI fold-increase on stimulated platelets in } \\
\text { PRP vs. resting platelets, median }\left[\mathrm{IQ}^{25-75}\right]\end{array}$} & CRP $10 \mu \mathrm{g} / \mathrm{mL}$ & $23^{[12-38]}$ & $26^{[15-49]}$ & 0.56 \\
\hline & TRAP $50 \mu \mathrm{M}$ & $4^{[2-12]}$ & $16^{[4-23]}$ & 0.07 \\
\hline \multirow{2}{*}{$\begin{array}{l}\text { CD63 MFI fold-increase on stimulated platelets in } \\
\text { PRP vs. resting platelets }\end{array}$} & CRP $10 \mu \mathrm{g} / \mathrm{mL}$ & $14^{[8-19]}$ & $18^{[10-22]}$ & 0.20 \\
\hline & TRAP $50 \mu \mathrm{M}$ & $4^{[2-8]}$ & $7^{[2-13]}$ & 0.48 \\
\hline
\end{tabular}

Abbreviations: ADP, adenosine diphosphate; AS, aortic valve stenosis; CAD, coronary artery disease; CRP, collagen-related peptide; MFI, median fluorescence intensity; PRP, platelet-rich plasma; SD, standard deviation; TAVI, transcatheter aortic valve implantation; TRAP, thrombin receptor activating peptide.

Note: The results of the two groups were compared using the non-parametric Mann-Whitney test.

microparticles $^{19-21}$ and platelet-monocyte aggregates ${ }^{22}$ in whole blood have been reported in these patients. Platelet generation of eicosanoids resulting from the oxidative transformation of arachidonic acid through cyclooxygenase 1 (COX-1) and lipoxygenase (LOX) pathways is an early response to platelet activation. ${ }^{23}$ The development of lipi- domics for sensitive and accurate quantitation of a large set of eicosanoids introduces potential new biomarkers of platelet activation, investigated for the first time in AS in our study. As all patients in both groups received low-dose aspirin, COX-1 products were not measurable and the flux of arachidonic acid was redirected to LOXs, mainly 12-LOX,
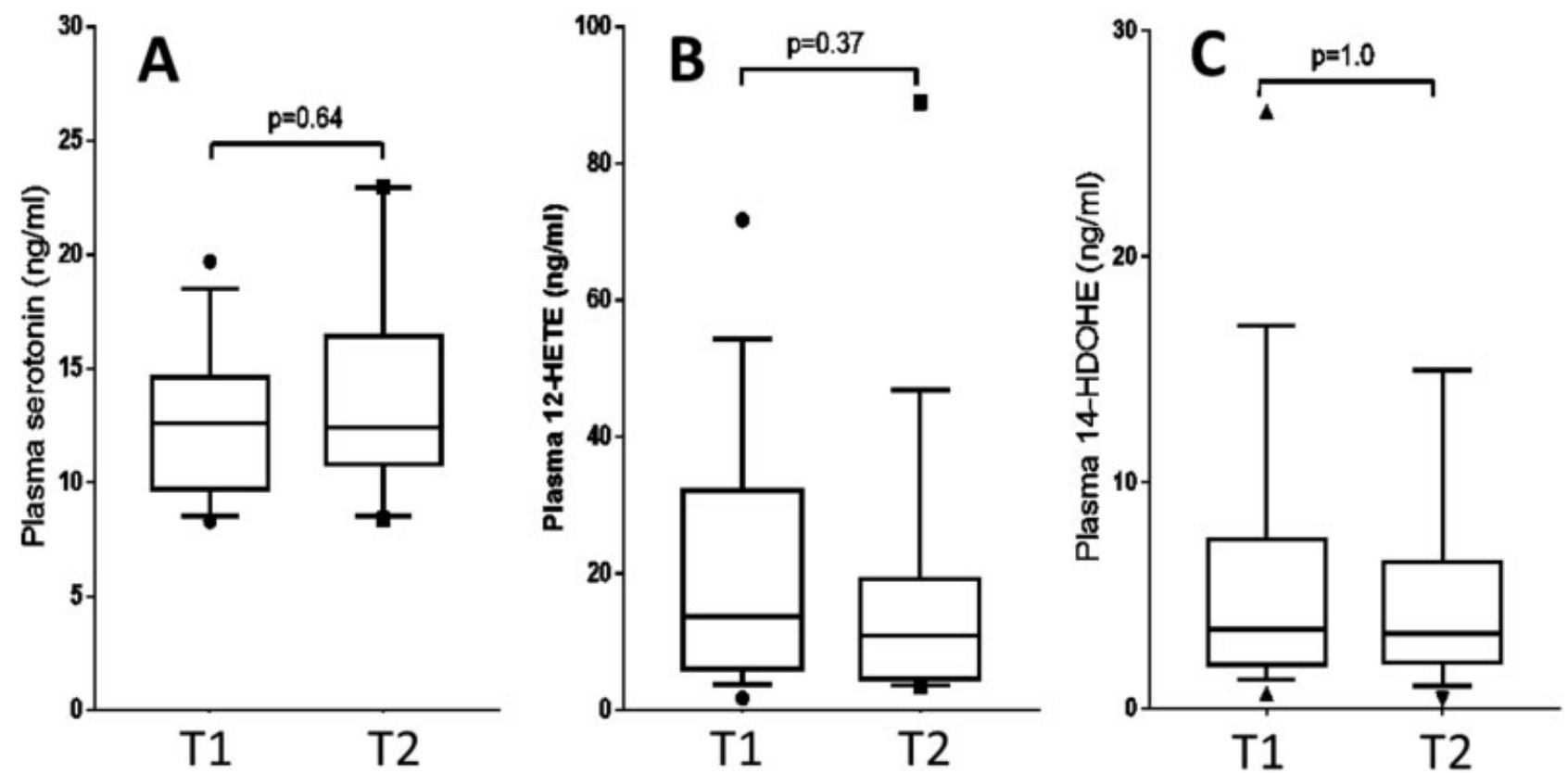

Fig. 1 Platelet activation markers in aortic blood sampled immediately before (T1) and 10-15 minutes (T2) after the placement of the aortic bio prosthesis. Whisker boxes: $10-25-75-90$ th percentiles and outliers. Statistical significance calculated using nonparametric paired Wilcoxon's test. 
the most abundant in platelets. ${ }^{23}$ The two main products released in plasma were 12-HETE and 14-HDoHE. The variability of plasma 12-HETE in our cohort was relatively large, as reported in collagen-stimulated platelets of healthy volunteers before or after aspirin administration. ${ }^{24}$ Although lowdose aspirin has also been reported to affect 12-LOX and to partially reduce 12 -HETE production by collagen-stimulated platelets, ${ }^{24}$ the higher levels in patients with AS compared with those with CAD similarly treated by aspirin (- Table 2 ), which remains after adjustment for age, are indicative of in vivo platelet activation induced by the pathological shear stress.

Compared with peripheral venous blood, the eicosanoid levels in aortic blood before TAVI were not significantly different and platelets did not expose more CD62-P or CD63. This suggests that the subset of platelets activated by high shear through the valve is too small for being detected downstream by flow cytometry. As activated platelets are rapidly removed from the circulation or do not circulate as isolated cells, they cannot be detected either in peripheral blood, in contrast to soluble biomarkers that persist longer. In addition, systemic activation by various factors besides high shear stress, such as left ventricular dysfunction, advanced atherosclerosis, or low-grade inflammation, may be preeminent.

The main result of the study is the absence of detectable platelet activation per procedure in blood sampled close to the injured valve (-Fig. 1). Captured debris in filters deployed in large cerebral arteries during TAVI associate thrombotic material to tissue-derived debris dislodged from the degenerated valve or the aorta. ${ }^{4}$ Activated platelets associated with such mixed material would escape to flow cytometry analysis. However, an increase of soluble markers serotonin and lipoxins was expected in blood collected close to the site of platelet interactions with the crushed aortic valve, before their dilution in systemic circulation. Although we showed normal reactivity of circulating platelets to soluble agonists in static conditions ( - Table 2 ), the shedding of platelet membrane receptors GPIb $\alpha$ and GPVI induced by high shear, ${ }^{16,25}$ combined with the defect of high-molecularweight von Willebrand factor, ${ }^{26-28}$ low-dose aspirin treatment, and thrombin inhibition by heparin, may explain our unexpected negative results.

This study has several limitations: (1) The series is short. However, there is no trend of acute change before and immediately after the procedure close to the aortic valve. (2) Only one time point after implantation is available. This was constrained by technical/safety reasons. However, it is unlikely that the bioprosthesis would provide a complete coverage of the thrombogenic surface within minutes after implantation. (3) The sensitivity and specificity of the biomarkers may be insufficient. However, the convergent results obtained with secreted, synthesized, and surfacebound markers confirm a chronic platelet activation reported by others in patients with severe AS, but they do not suggest acute per procedure activation. (4) Only one model of self-expandable bioprosthesis was used in this study. Platelet activation dependent on the model has been reported, associated with residual aortic regurgitation. ${ }^{29}$ In our study, only $1 / 17$ patient had mild paravalvular leakage on TEE at discharge. (5) Finally, and most importantly, no patient developed a clinically apparent ischemic event after valve implantation. Our results do not exclude a contribution of platelets to such complication when it occurs.

Patients undergoing TAVI are at high risk for both ischemic stroke and bleeding, most of them occurring early after the procedure, but the risk of severe bleeding is approximately twice that of ischemic complications. A recent meta-analysis comparing monotherapy with aspirin (SAPT) to DAPT indicated that DATP was associated with a higher rate of major adverse events after TAVI, mainly driven by an increased risk of major or life-threatening bleeding complications along with a lack of beneficial effect on ischemic events. $^{30}$ In patients treated with DATP, low (vs. high) ontreatment platelet reactivity is associated with more bleeding events without difference in ischemic events at 30-day follow-up. ${ }^{31}$ In a recent study, no difference in the rates of ischemic complications was observed between patients who received a loading dose of clopidogrel, compared with those who did not. ${ }^{32}$ Although we did not compare patients under DAPT vs. SAPT, our results are consistent with these findings. They suggest that during uneventful TAVI on monotherapy with aspirin, acute per procedure platelet activation remains limited. However, due to the small sample size of the population, this analysis is a hypothesis-generating case series and thus the conclusions should be confirmed in a larger study.

\section{Funding}

This study was supported by a research grant from Medtronic and by the Academic Hospital of Toulouse. Patient selection, data collection and analysis, manuscript redaction, and submission were fully independent from the sponsor. B.P. is a senior member of the Institut Universitaire de France.

\section{Conflict of Interest}

Nicolas Dumonteil received proctors fees and is consultant for Edwards LifeScience, Medtronic, and Boston Scientific. Bertrand Marcheix received proctors fees from Edwards LifeScience and Medtronic. The other authors do not have any potential conflict of interest to disclose.

\section{Acknowledgments}

We thank the Clinical Research assistants Ludovic Lacassagne and Adeline Hupé of the Department of Cardiology. Cytometry analyses were performed on the cytometry core facility, INSERM U 1048, part of TRI Imaging platform (Genotoul).

\section{References}

1 Thonghong T, De Backer O, Søndergaard L. Comprehensive update on the new indications for transcatheter aortic valve replacement in the latest 2017 European guidelines for the management of valvular heart disease. Open Heart 2018;5(01):e000753 
2 Gaede L, Blumenstein J, Liebetrau C, et al. Outcome after transvascular transcatheter aortic valve implantation in 2016. Eur Heart J 2018;39(08):667-675

3 Nijenhuis VJ, Bennaghmouch N, van Kuijk JP, Capodanno D, ten Berg JM. Antithrombotic treatment in patients undergoing transcatheter aortic valve implantation (TAVI). Thromb Haemost 2015; 113(04):674-685

4 Puri R, Altisent OA, Campelo-Parada F, del Trigo M, Regueiro A, Rodés-Cabau J. Balancing the risks of thrombosis and bleeding following transcatheter aortic valve implantation: current stateof-evidence. Curr Pharm Des 2016;22(13):1904-1910

5 Erdoes G, Basciani R, Huber C, et al. Transcranial Doppler-detected cerebral embolic load during transcatheter aortic valve implantation. Eur J Cardiothorac Surg 2012;41(04):778-783, discussion 783-784

6 Van Mieghem NM, El Faquir N, Rahhab Z, et al. Incidence and predictors of debris embolizing to the brain during transcatheter aortic valve implantation. JACC Cardiovasc Interv 2015;8(05): 718-724

7 Gandhi S, Schwalm J-DR, Velianou JL, Natarajan MK, Farkouh ME. Comparison of dual-antiplatelet therapy to mono-antiplatelet therapy after transcatheter aortic valve implantation: systematic review and meta-analysis. Can J Cardiol 2015;31(06):775-784

8 Vahanian A, Alfieri O, Andreotti F, et al; Joint Task Force on the Management of Valvular Heart Disease of the European Society of Cardiology (ESC); European Association for Cardio-Thoracic Surgery (EACTS). Guidelines on the management of valvular heart disease (version 2012). Eur Heart J 2012;33(19):2451-2496

9 Nishimura RA, Otto CM, Bonow RO, et al; ACC/AHA Task Force Members. 2014 AHA/ACC Guideline for the Management of Patients with Valvular Heart Disease: a report of the American College of Cardiology/American Heart Association Task Force on Practice Guidelines. Circulation 2014;129(23):e521-e643

10 Tchetche D, Dumonteil N, Sauguet A, et al. Thirty-day outcome and vascular complications after transarterial aortic valve implantation using both Edwards Sapien and Medtronic CoreValve bioprostheses in a mixed population. EuroIntervention 2010;5(06):659-665

11 Leon MB, Piazza N, Nikolsky E, et al. Standardized endpoint definitions for transcatheter aortic valve implantation clinical trials: a consensus report from the Valve Academic Research Consortium. Eur Heart J 2011;32(02):205-217

12 Le Faouder P, Baillif V, Spreadbury I, et al. LC-MS/MS method for rapid and concomitant quantification of pro-inflammatory and pro-resolving polyunsaturated fatty acid metabolites. J Chromatogr B Analyt Technol Biomed Life Sci 2013;932:123-133

13 Xiao Z, Théroux P. Platelet activation with unfractionated heparin at therapeutic concentrations and comparisons with a low-molecular-weight heparin and with a direct thrombin inhibitor. Circulation 1998;97(03):251-256

14 Natorska J, Bykowska K, Hlawaty M, Marek G, Sadowski J, Undas A. Increased thrombin generation and platelet activation are associated with deficiency in high molecular weight multimers of von Willebrand factor in patients with moderate-to-severe aortic stenosis. Heart 2011;97(24):2023-2028

15 Wurster T, Tegtmeyer R, Borst 0 , et al. Platelet expression of stromal cell-derived factor- 1 is associated with the degree of valvular aortic stenosis. PLoS One 2014;9(05):e97405
16 Lukito P, Wong A, Jing J, et al. Mechanical circulatory support is associated with loss of platelet receptors glycoprotein Ib $\alpha$ and glycoprotein VI. J Thromb Haemost 2016;14(11):2253-2260

17 Motovska Z, Odvodyova D, Karpisek M, et al. Platelet-derived chemokines, PF-4 and RANTES, are significantly increased in hemodynamically significant degenerative aortic stenosis. Int J Cardiol 2011;152(02):273-275

18 Rouzaud-Laborde C, Delmas C, Pizzinat N, et al. Platelet activation and arterial peripheral serotonin turnover in cardiac remodeling associated to aortic stenosis. Am J Hematol 2015;90(01): 15-19

19 Diehl P, Nagy F, Sossong V, et al. Increased levels of circulating microparticles in patients with severe aortic valve stenosis. Thromb Haemost 2008;99(04):711-719

20 Jung C, Lichtenauer M, Figulla HR, et al. Microparticles in patients undergoing transcatheter aortic valve implantation (TAVI). Heart Vessels 2017;32(04):458-466

21 Marchini JF, Miyakawa AA, Tarasoutchi F, Krieger JE, Lemos P, Croce $\mathrm{K}$. Endothelial, platelet, and macrophage microparticle levels do not change acutely following transcatheter aortic valve replacement. J Negat Results Biomed 2016;15:7

22 Badr Eslam R, Gremmel T, Schneller A, et al. High levels of plateletmonocyte aggregates after valve replacement for aortic stenosis: relation to soluble P-selectin and P-selectin glycoprotein ligand-1 genes. Thromb Res 2012;129(04):453-458

23 O'Donnell VB, Murphy RC, Watson SP. Platelet lipidomics: modern day perspective on lipid discovery and characterization in platelets. Circ Res 2014;114(07):1185-1203

24 Maskrey BH, Rushworth GF, Law MH, et al. 12-hydroxyeicosatetraenoic acid is associated with variability in aspirin-induced platelet inhibition. J Inflamm (Lond) 2014;11(01):33

25 Chen Z, Mondal NK, Zheng S, et al. High shear induces platelet dysfunction leading to enhanced thrombotic propensity and diminished hemostatic capacity. Platelets 2019;30(01):112-119

26 Panzer S, Badr Eslam R, Schneller A, et al. Loss of high-molecularweight von Willebrand factor multimers mainly affects platelet aggregation in patients with aortic stenosis. Thromb Haemost 2010;103(02):408-414

27 Van Belle E, Rauch A, Vincentelli A, et al. Von Willebrand factor as a biological sensor of blood flow to monitor percutaneous aortic valve interventions. Circ Res 2015;116(07):1193-1201

28 Takahashi N, Tanabe K, Yoshitomi H, et al. Impairment of platelet retention rate in patients with severe aortic valve stenosis. J Cardiol 2013;62(03):171-175

29 Fateh-Moghadam S, Voesch S, Htun P, et al. Platelet activation is less enhanced in the new balloon expandable Edwards Sapien 3 valve compared to its predecessor model (Edwards Sapien XT). Thromb Haemost 2016;115(01):109-116

30 Maes F, Stabile E, Ussia GP, et al. Meta-analysis comparing single versus dual antiplatelet therapy following transcatheter aortic valve implantation. Am J Cardiol 2018;122(02):310-315

31 Gross L, Jochheim D, Nitschke T, et al. Platelet reactivity and early outcomes after transfemoral aortic valve implantation. Thromb Haemost 2018;118(10):1832-1838

32 Nijenhuis VJ, Ten Berg JM, Hengstenberg C, et al. Usefulness of clopidogrel loading in patients who underwent transcatheter aortic valve implantation (from the BRAVO-3 Randomized Trial). Am J Cardiol 2019;123(09):1494-1500 